\title{
Droplet formation of a thermal sideshooter inkjet printhead
}

\author{
Ping-Hei Chen *, Wen-Cheng Chen, Pei-Pei Ding, S.H. Chang \\ Department of Mechanical Engineering, National Taiwan University, No. 1, Sec. 4, Roosevelt Rd., Taipei, Taiwan \\ Received 14 November 1997; accepted 2 April 1998
}

\begin{abstract}
The present study aims to provide numerical predictions on the droplet formation process for a thermal sideshooter inkjet printhead, which directly affects the printing quality of the printhead. The calculation procedure starts from the heat up of the printhead by an input electrical pulse, then obtains the pressure impulse response curve due to the formation and collapse of a bubble in the ink, the ink flow motion in the ink nozzle, and finally the evolution process of the ejected ink droplet outside the ink nozzle. The governing continuity and momentum equations for the flow motion in the ejected ink droplet are solved on the Eulerian frame by an implicit finite-difference scheme. The ink droplet leading is then updated to the new location from the newly obtained velocity. A comparison is made between the present numerical predictions with experimental results. A good agreement has been found. In addition, the present study shows the effects of the operating voltage of electrical pulse, the ink properties, and the gravity on the breakoff time of ejected droplet from the ink nozzle exit and the separation time of the long tail from main droplet. (C) 1998 Elsevier Science Inc. All rights reserved.
\end{abstract}

Keywords: Inkjet printhead; Ink drop formation; Surface tension; Two-phase flow

\section{Notation}

$\bar{A}$

$\bar{B}$

$\bar{B}$

$a$

E

$\mathrm{Fr}$

$\mathrm{g}_{z}$
$h_{f g}$

$K$

$P$

$P_{\text {air }}$

$P_{\text {r }}$

$P_{\mathrm{s}}$

\section{$P_{\mathrm{t}}$}

$P_{\mathrm{v}}$

$R$

$\mathrm{Re}$

$r$

$r_{\mathrm{i}}$

$r_{\mathrm{N}}$ dimensionless inertial factor for the ink flow motion in the nozzle dimensionless frictional factor for the ink flow motion in the nozzle radius of the ink nozzle exit, $\mu \mathrm{m}$ operating voltage of electrical impulse, $\mathrm{V}$ Froude number, $=\left(T / \rho g_{z} a^{2}\right)^{1 / 2}$ gravitational acceleration along the $z$ axis, $\mu \mathrm{m} / \mu \mathrm{s}^{2}$ heat of vaporization, $\mathrm{J} \mathrm{kg}^{-1}$ universal gas constant, $\mathrm{J} \mathrm{mol}^{-1} \mathrm{~K}^{-1}$ pressure, $\mu \mathrm{N} / \mu \mathrm{m}^{2}$

\section{surrounding air pressure, $\mu \mathrm{N} / \mu \mathrm{m}^{2}$} relative pressure between ink and surrounding air, $\mu \mathrm{N} / \mu \mathrm{m}^{2}$

$P_{\mathrm{s}} \quad$ saturated vapor pressure at surrounding air temperature, $\mu \mathrm{N} / \mu \mathrm{m}^{2}$

$$
\text { saturated vapor pressure at the threshold tempera- }
$$
ture of homogeneous nucleation, $\mu \mathrm{N} / \mu \mathrm{m}^{2}$

vapor pressure in the bubble, $\mu \mathrm{N} / \mu \mathrm{m}^{2}$

meniscus radius of ink droplet on the cross section normal to the nozzle axis, $\mu \mathrm{m}$

Reynolds number, $=\rho V_{0} a / \mu$

radial coordinate, $\mu \mathrm{m}$

radius of the nozzle cross-section at the bubble-ink interface, $\mu \mathrm{m}$

principal radius of ink-gas interface on the plane containing the nozzle axis, $\mu \mathrm{m}$

\footnotetext{
${ }^{*}$ Corresponding author. E-mail: phchen@ccms.ntu.edu.tw.
}

principal radius of ink-gas interface on the plane normal to the nozzle axis, $\mu \mathrm{m}$

surface tension coefficient, $\mu \mathrm{N} / \mu \mathrm{m}$

temperature at the boiling point of ink, $\mathrm{K}$

temperature of vapor bubble, $\mathrm{K}$

time, $\mu$ s

time instant when the vapor bubble starts to form, $\mu \mathrm{s}$ break-off time of the ejected ink droplet from the nozzle exit, $\mu$ s

separation time of the long tail from the main droplet, $\mu$ s

axial velocity, $\mu \mathrm{m} / \mu \mathrm{s}$

volume percentage of ink in the nozzle, $\%$

width of the applied electrical pulse, $\mu$ s molecular weight of the vapor in the bubble, $\mathrm{kg}$ $\mathrm{mol}^{-1}$

Weber number, $=\rho V_{0}^{2} a / T$

axial coordinate originated from the nozzle exit, $\mu \mathrm{m}$

$z$-coordinate value of the bubble-ink interface, $\mu \mathrm{m}$ distance from the tail of broken-off droplet to the nozzle exit, $\mu \mathrm{m}$

distance from the leading edge of ink droplet to the nozzle exit, $\mu \mathrm{m}$

empirical constant in Eq. (24)

dynamic viscosity, $\mathrm{mg} /(\mu \mathrm{m} \cdot \mu \mathrm{s})$

ink density, $\mathrm{mg} / \mu \mathrm{m}^{3}$

normal stress acting on the ink element, $\mu \mathrm{N} / \mu \mathrm{m}^{2}$ 
$\tau \quad$ time constant of vapor bubble pressure decay in Eq. (24), $\mu \mathrm{s}$

$\zeta$ axial coordinate on the computational domain

\section{Superscript \\ $n \quad$ current time step index \\ $n+1 \quad$ next time step index \\ - dimensionless parameter}

$\begin{array}{ll}\text { Subscript } \\ \text { air } & \text { air } \\ \text { exit } & \text { nozzle exit } \\ \mathrm{i} & \text { index of grid node } \\ \text { ink } & \begin{array}{l}\text { ink } \\ \text { nodal index for the leading edge of ejected ink } \\ \mathrm{N}\end{array} \\ & \begin{array}{l}\text { droplet } \\ \text { characteristic parameter } \\ \text { nodal index for the trailing edge of ejected ink } \\ \text { droplet }\end{array}\end{array}$

\section{Introduction}

In recent years, the inkjet printer has become the most dominant output device used with the personal computer for the color printing due to its low cost and comparable printing quality. However, the printing quality for the inkjet printer is closely related to the ink droplet formation process which can be affected by the ink property, the pressure impulse history, and the ink flow passage in the printhead. Therefore, it is essential to predict the temporal evolution process of ejected ink droplet in order to improve the printing quality.

Two different types of printhead, piezoelectric and thermal bubble, are commonly available in the market. The present study only focuses on the droplet ejection process of a thermal bubble inkjet printhead. Based on the directions of bubble growth and ejected ink flow, the thermal bubble inkjet printhead can be further classified into three categories (Krause et al., 1996)., The ejected ink flow direction is perpendicular with the bubble growth direction for the edgeshooter printhead. For a sideshooter printhead, the ink droplet moves in the same direction as that of the vapor bubble growth. However, the moving direction of ink droplet is opposite to that of vapor bubble growth for a backshooter printhead. There is no surprise that few reports are available for showing the droplet formation process of the inkjet printhead due to the strong competition among the leading manufacturing companies. Thus, detailed configuration of printhead will not be revealed from most companies. Fromm (1984) presented the droplet formation ejected from a circular nozzle with a constant cross section by employing a marker-and-cell scheme to solve the two-dimensional Navier-Stokes equations. However, the driving pressure response curve at the nozzle inlet was simplified by a square pulse in his study. With the same nozzle and the driving pressure response curve, Adams and Roy (1986) used a simplified one-dimensional model to describe the droplet formation process. They employed a MacCormack's predictorcorrector finite-difference scheme to solve the governing equations on the Lagrangian coordinate. The comparison indicates a good agreement on predicted results between one-dimensional model in Adams and Roy (1986) and two-dimensional model in Fromm (1984). Therefore, a less-complex one-dimensional model in present study can be used to provide good qualitative results for predicting the temporal evolution of ejected ink droplet of the sideshooter thermal bubble printhead.

For edgeshooter printhead, Asai et al. (1987) predicted the time-varying temperature distribution in the multilayer thin film structure with a one-dimensional heat conduction model. The driving pressure history with the bubble formation and collapse was then obtained from the Clapeyron-Clausius equation and the energy balance between the ink and air bubble. With the rising pressure in the bubble as the driving force, the ink flow motion in the nozzle was described by the one-dimensional hydraulic equation. The ejected droplet length can be easily evaluated from the one-dimensional hydraulic equation. A good agreement has been illustrated between the predicted and measured ejected droplet length. Furthermore, Asai (1992) solved the complete three-dimensional NavierStokes equations to obtain the droplet formation process for a edgeshooter printhead.

Chen et al. (1997a) employed the same model as the one in Asai et al. (1987) to obtain the ejected droplet length for a sideshooter printhead, as shown in Fig. 1. Both numerical and experimental data were presented in their studies. However, the predicted and measured ejected droplet lengths agreed only in the initial period of time after the electrical heating impulse was applied because the surface tension of droplet meniscus was neglected.

To the author's knowledge, few numerical or experimental results have been provided about the droplet formation process for a sideshooter printhead in prior studies. The present study aims to show a complete droplet ejection cycle for this particular type of thermal inkjet printhead under the application of heating electrical impulse to the separation of a long tail droplet from the ejected main droplet. Detailed configuration of the printhead is illustrated in Fig. 1. The operating procedure of the thermal bubble inkjet printhead can be simply described as follows. An electrical square pulse was applied to the resistor layer of the multilayer thin film structure of the printhead through the electrode layer. The typical electric pulse has a pulse width ranging from 2 to $7 \mu$ s and an operat-

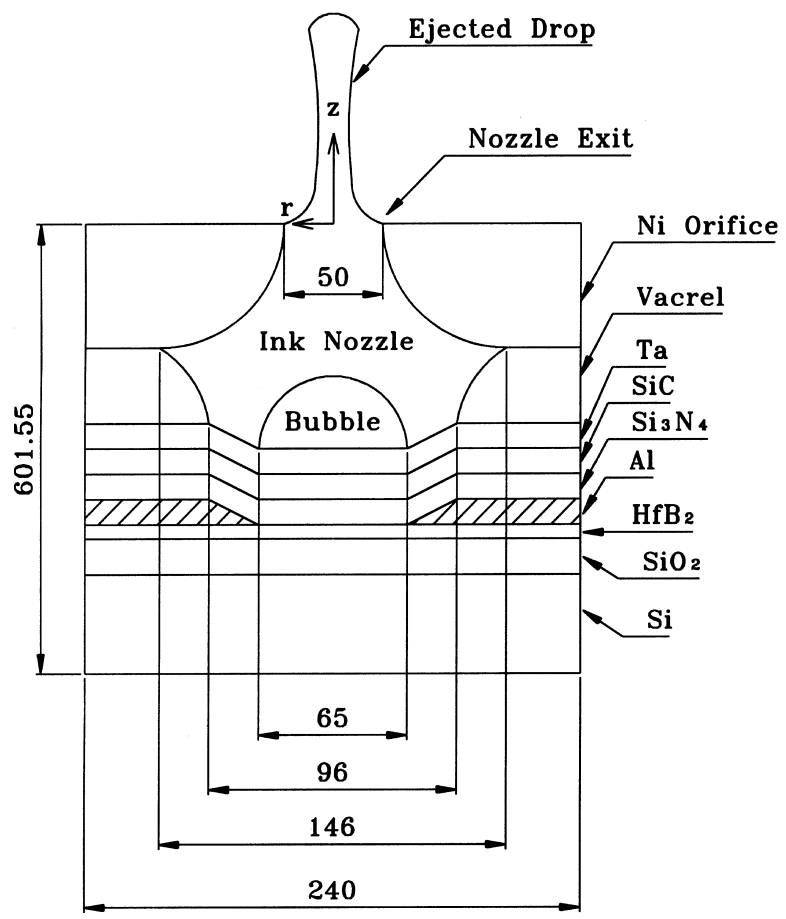

Fig. 1. Geometry and dimensions of test printhead: Ta passivation layer thickness $0.5 \mu \mathrm{m} ; \mathrm{SiC}$ and $\mathrm{Si}_{3} \mathrm{~N}_{4}$ overcoat layer thickness $0.25 \mu \mathrm{m}$ and $0.5 \mu \mathrm{m}$ respectively; $\mathrm{HfB}_{2}$ resistor layer thickness $0.1 \mu \mathrm{m}$; $\mathrm{Al}$ electrode layer thickness $0.5 \mu \mathrm{m} ; \mathrm{SiO}_{2}$ thermal insulation layer thickness $1.7 \mu \mathrm{m}$ and Si substrate layer thickness $525 \mu \mathrm{m}$. 
ing voltage from 16 to $23 \mathrm{~V}$. Large heat flow which was generated in the resistor layer was transmitted into substrate and ink through heat conduction process. The temperature of ink at the ink-solid interface was immediately raised to a value about the critical temperature within few $\mu$ s. Combined with the extremely large heat flux into the ink, the homogeneous nucleation occurred in the ink. A vapor bubble whose pressure was expected to be extremely high was then formed above the passivation layer. This high pressure vapor bubble became the driving force to push the ink out of the nozzle. Once the bubble was formed, heat energy was cut off from the heating layer but dissipated from the heated ink into the bubble for the evaporation of ink. Consequently, the vapor bubble continues to grow up to a maximum volume and then collapses. Without the blockage of bubble on the ink inlet, the nozzle was then refilled with ink. The sharp pressure drop in the vapor bubble is unable to provide enough ink into the droplet and then resulted in the separation of ejected ink from the ink nozzle exit. The ejected ink droplet usually had a comet shape with a long tail. During the travel of droplet in the air, two satellite droplets and one long tail could form and separate sequentially from the main ink droplet due to the effects of viscous force and surface tension of meniscus. To improve the printing quality, it becomes important to predict the time when the separation of main droplet from the nozzle exit and the formation of ink satellite and long tail occurs. Otherwise, blur can occur on the paper when the ink droplet impinges on the surface of paper.

To have the complete cycle of ink formation process, the driving pressure response curve was obtained from the model used in the work of Asai (1991) and Chen et al. (1997a). This pressure response curve is further used to predict the ink flow motion in the nozzle. After the ink is ejected from the nozzle, an implicit finite-difference scheme on the Eulerian frame was employed to solve a simplified one-dimensional model (Adams and Roy, 1986) for obtaining the local ink flow motion in the ejected ink droplet and the meniscus radius of droplet for a sideshooter printhead. After the ink velocity is obtained, the leading edge of droplets is then updated to the new location for the next time step. To validate our predicted results, the predicted ink droplet formation process is also compared with the measured results presented in Chen et al. (1997b). The present study also investigates the effects of operating voltage, properties of ink, and gravity on droplet formation process, the break-off time of ink droplet from the nozzle exit, and the separation time of the long tail from main droplet.

\section{Governing equations and numerical methods}

Fig. 1 shows the schematic view of an ejected ink droplet from the nozzle of a printhead and Fig. 2 illustrates the control volume that is chosen to derive the momentum equation for an ink fluid element of the ink droplet. Assumptions in this study are given as follows: (1) the ink flow is axisymmetric, (2) the axial velocity and normal stress over the cross section are uniform, (3) the ink is non-wetting in the nozzle, and (4) the ink flow is incompressible. Then, the one-dimensional continuity and momentum equations for the flow motion in the ink droplet, which are same as the ones used by Adams and Roy (1986) can be expressed as

$$
\begin{aligned}
& \frac{\partial V}{\partial t}+V \frac{\partial V}{\partial z}=\frac{1}{\rho} \frac{\partial \sigma_{z z}}{\partial z}+g_{z}, \\
& \frac{\partial R^{2}}{\partial t}+\frac{\partial}{\partial z}\left(R^{2} V\right)=0
\end{aligned}
$$

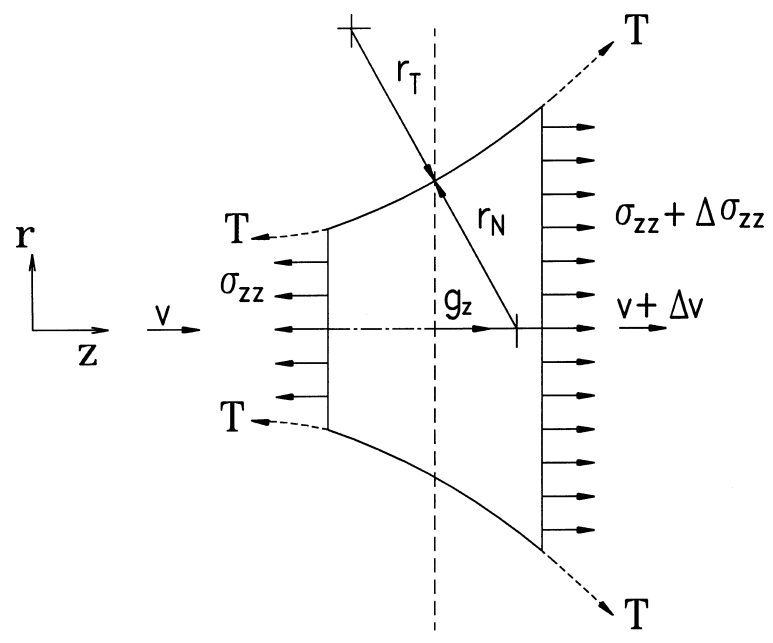

Fig. 2. Control volume of an ink element in the ejected droplet.

where $V$ is the axial velocity, $R(z, t)$ is the meniscus radius of droplet on the cross section normal to the ink flow motion, $g_{z}$ is the gravitational acceleration along $z$ direction, and $\rho$ is the ink density. In Eq. (1), $\sigma_{z z}$ is the total normal stress exerted on the ink element, given by

$\sigma_{z z}=-P_{\text {ink }}+2 \mu \frac{\partial V}{\partial z}$,

where $\mu$ and $P_{\text {ink }}$ denote the ink viscosity and pressure, respectively. Over the ink-air interface, the surface tension plays an important role on the droplet formation. To account for the surface tension effect, the ink pressure can be further expressed as

$P_{\text {ink }}-P_{\text {air }}=P_{\mathrm{r}}=T\left(\frac{1}{r_{N}}+\frac{1}{r_{T}}\right)-\mu \frac{\partial V}{\partial z}$,

where $T$ denotes the surface tension coefficient of ink. In Eq. (4), $r_{\mathrm{N}}$ and $r_{\mathrm{T}}$, as shown in Fig. 2, are two principal radii of curvature over the free surface of ink droplet. Both $r_{\mathrm{N}}$ and $r_{\mathrm{T}}$ are defined respectively as

$r_{\mathrm{N}}=R\left[1+\left(\frac{\partial R}{\partial z}\right)^{2}\right]^{1 / 2}$

$r_{\mathrm{T}}=-\left[1+\left(\frac{\partial R}{\partial z}\right)^{2}\right]^{3 / 2} / \frac{\partial^{2} R}{\partial z^{2}}$.

Substituting Eq. (4) into Eq. (3) yields

$\sigma_{z z}=-T\left(\frac{1}{r_{N}}+\frac{1}{r_{T}}\right)+3 \mu \frac{\partial V}{\partial z}$.

Now, Eq. (1) can be rearranged with the new expression of $\sigma_{z z}$ as

$\frac{\partial V}{\partial t}+V \frac{\partial V}{\partial z}=\frac{1}{\rho}\left[-T \frac{\partial}{\partial z}\left(\frac{1}{r_{N}}+\frac{1}{r_{T}}\right)+3 \mu \frac{\partial^{2} V}{\partial z^{2}}\right]+g_{z}$.

To express both continuity and momentum equations in the non-dimensional forms, the following non-dimensional variables are introduced

$$
\begin{aligned}
& \bar{t}=\frac{t}{t_{0}}, \quad \bar{z}=\frac{z}{z_{0}}, \quad \bar{V}=\frac{V}{V_{0}}, \quad \bar{R}=\frac{R}{a}, \quad \bar{r}_{\mathrm{N}}=\frac{r_{\mathrm{N}}}{a}, \\
& \bar{r}_{\mathrm{T}}=\frac{r_{\mathrm{T}}}{a},
\end{aligned}
$$

where the characteristic velocity and time are defined respectively as 
$V_{0}=\left(\frac{T}{\rho a}\right)^{1 / 2}$,

$t_{0}=\frac{a}{V_{0}}=\left(\frac{\rho a^{3}}{T}\right)^{1 / 2}$.

In both Eq. (10) and Eq. (11), $a$ is the radius of nozzle exit. With these non-dimensional variables, Eq. (1) and Eq. (8) become

$$
\begin{aligned}
& \frac{\partial \bar{R}^{2}}{\partial \bar{t}}+\frac{\partial\left(\bar{R}^{2} \bar{V}\right)}{\partial \bar{z}}=0, \\
& \frac{\partial \bar{V}}{\partial \bar{t}}+\bar{V} \frac{\partial \bar{V}}{\partial \bar{z}}=\left[-\frac{\partial}{\partial \bar{z}}\left(\frac{1}{\bar{r}_{\mathrm{N}}}+\frac{1}{\bar{r}_{\mathrm{T}}}\right)+3 \frac{\mathrm{We}}{\operatorname{Re}} \frac{\partial^{2} \bar{V}}{\partial \bar{z}^{2}}\right]+\frac{1}{\mathrm{Fr}^{2}},
\end{aligned}
$$

where the ratio of Weber number to Reynolds number, and the Froude number are defined respectively as

$$
\begin{aligned}
& \frac{\mathrm{We}}{\mathrm{Re}}=\frac{\mu}{(\rho a T)^{1 / 2}}, \\
& \mathrm{Fr}=\left(\frac{T}{\rho g_{z} a^{2}}\right)^{1 / 2} .
\end{aligned}
$$

To solve Eqs. (12) and (13), initial and boundary conditions should be properly given. At $\bar{t}=0$, it is reasonable to assume $\bar{R}=1$ and $\bar{V}=0$. However, the boundary conditions for the ink droplet can be different before and after the break-off of the droplet from the nozzle exit during the droplet formation process. The boundary conditions are given by:

$$
\left.\begin{array}{ll}
\bar{V}=\bar{V}_{\text {exit }}, & \text { at } \bar{z}=0 \\
\frac{\partial \bar{V}}{\partial \bar{z}}=0, & \bar{R}=0, \text { at } \bar{z}=\bar{z}_{\text {tip }}
\end{array}\right\} \text { before the break-off of }
$$

droplet

and

$$
\left.\begin{array}{ll}
\frac{\partial \bar{V}}{\partial \bar{z}}=0, & \text { at } \bar{z}=\bar{z}_{\text {tail }} \\
\frac{\partial \bar{V}}{\partial \bar{z}}=0, & \bar{R}=0, \text { at } \bar{z}=\bar{z}_{\text {zip }}
\end{array}\right\} \text { after the break-off of }
$$

droplet.

In Eq. (16), the ejected ink velocity at the nozzle exit, $\bar{V}_{\text {exit }}$, can be determined by solving the one-dimensional hydraulic equation, expressed as

$\bar{A} \frac{\mathrm{d} \bar{V}_{\text {exit }}}{\mathrm{d} \bar{t}}+\frac{\mathrm{We}}{\operatorname{Re}} \bar{B} \bar{V}_{\text {exit }}=\bar{P}_{\mathrm{v}}-\bar{P}_{\text {air }}-\left(\frac{1}{\bar{r}_{N}}+\frac{1}{\bar{r}_{T}}\right)_{\text {exit }}$,

where $\bar{P}_{\mathrm{v}}$ is the dimensionless vapor pressure in the bubble, $\bar{P}_{\text {air }}$ is the dimensionless surrounding air pressure, and $\bar{A}$ and $\bar{B}$ are the dimensionless inertial factor and frictional factor, respectively for the ink flow motion in the nozzle. These aforementioned dimensionless parameters are defined as

$\bar{A}=\frac{r_{\mathrm{i}}^{2}}{a} \int_{z_{\mathrm{i}}(t)}^{0} r^{-2} \mathrm{~d} z$

$\bar{B}=8 a^{3} \int_{z_{\mathrm{i}}(t)}^{0} r^{-4} \mathrm{~d} z$

$\bar{P}_{\mathrm{v}}=\frac{P_{\bar{v}}}{\rho V_{0}^{2}}, \quad \bar{P}_{\text {air }}=\frac{P_{\text {air }}}{\rho V_{0}^{2}}$,

where $r_{\mathrm{i}}$ denotes the radius of the nozzle cross-section at the bubble-ink interface and $z_{\mathrm{i}}$ is the $z$-coordinate of the interface. The detailed nozzle profiles are given in the paper of Chen et al. (1997a). Furthermore, Eq. (18) is subject to the initial conditions:

$$
\bar{V}_{\text {exit }}(\bar{t})=0 \text { at } \bar{t}=\bar{t}_{b}
$$

where $\bar{t}_{b}$ denotes the time instant when the vapor bubble starts to form at the interface of ink and the passivation layer.

In present study the process of bubble growth is classified into two stages for prediction of vapor pressure as the works of Asai (1991) and Chen et al. (1997a). In the early stage after the electrical impulse was applied, the Clausius-Clapeyron equation is used to describe the vapor pressure, given by

$P_{\mathrm{v}}=P_{\text {air }} \exp \left[\frac{w h_{f g}}{K}\left(\frac{1}{T_{\mathrm{bp}}}-\frac{1}{T_{\mathrm{v}}}\right)\right]$,

where $w$ is the molecular weight of the vapor, $K$ is the universal gas constant, $T_{\mathrm{v}}$ denotes the temperature of vapor bubble, and $h_{f g}$ and $T_{b p}$ are the heat of vaporization and boiling point temperature of ink at the surrounding air pressure, respectively. In the later stage, a first-order exponential decay formula is used to approximate the vapor pressure drop, given as

$P_{\mathrm{v}}=\left(P_{\mathrm{t}}-P_{\mathrm{s}}\right) \exp \left[-\left(\frac{t-t_{\mathrm{b}}}{\tau}\right)^{\lambda}\right]+P_{\mathrm{s}}$,

where $\tau$ is a time constant, $P_{\mathrm{t}}$ and $P_{\mathrm{s}}$ denote the values for saturated vapor pressure at the threshold temperature of homogeneous nucleation and surrounding air temperature, respectively. The detailed derivation for obtaining the threshold temperature of homogeneous nucleation and the temperature of vapor bubble in Eq. (23) can be found in the work of Chen et al. (1997a). In Eq. (24), the $\lambda$ value is carefully selected by matching the time histories between the experimental and simulated ejected ink lengths. In this study, a same $\lambda$ value of 0.32 for different applied electrical impulses was chosen. However, the criterion for dividing the process of bubble growth into early and later stage is the heat flux transferred from the heated ink into the bubble reaches the maximum value. At a given $\lambda$ value, the time constant $\tau$ is solved by letting the obtained vapor pressure values in Eq. (23) and Eq. (24) be equal at the instant of the maximum heat flux.

Fig. 3(a) and Fig. 3(b) show the typical vapor pressure response curves and the volume percentages of ink in the nozzle under different operating voltages at $W=3 \mu$ s for the present test printhead, respectively. A smaller volume percentage occupied by the ink in the nozzle indicates a greater vapor bubble volume. However, the threshold temperature of homogeneous nucleation is higher for the electrical pulse with a larger operating voltage. It makes the larger operating voltage has higher initial vapor pressure as shown in Fig. 3(a). After the homogeneous nucleation in the ink, an extremely high pressure, usually greater than $10.0 \mathrm{MPa}$, occurs in the vapor bubble. Once the bubble is formed, the heat energy is no longer transferred from the resistor layer through the bubble into the ink because of an extremely low thermal conductivity of vapor. The energy stored in the ink that is transferred from the resistor layer before the incipience of homogeneous nucleation, becomes the only heat source to vaporize the liquid ink for the continuous growth of the vapor bubble. It can be expected that a sharp decrease in temperature of vapor bubble accompany with the growing bubble will result the sharp drop of vapor pressure history. For a higher operating voltage of electrical pulse, the incipience of homogeneous nucleation occurs earlier, thus less heat energy is stored in the ink. Consequently, the volume of vapor bubble formed will be smaller. Therefore, it is expected that more ink (a lower minimum value in the volume percentage curve) will be driven out of the nozzle by a lower operating voltage at $W=3 \mu \mathrm{s}$.

Note that the ejected ink velocity at the nozzle exit can not be obtained without knowing the curvatures of ink droplet at the nozzle exit. Thus, Eq. (18) is coupled with Eq. (12) and Eq. (13) and solved through iteration. Once the ink droplet 


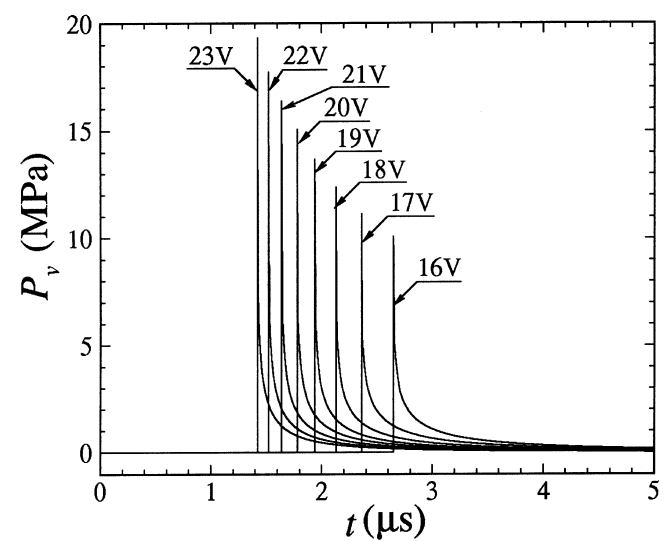

(a)

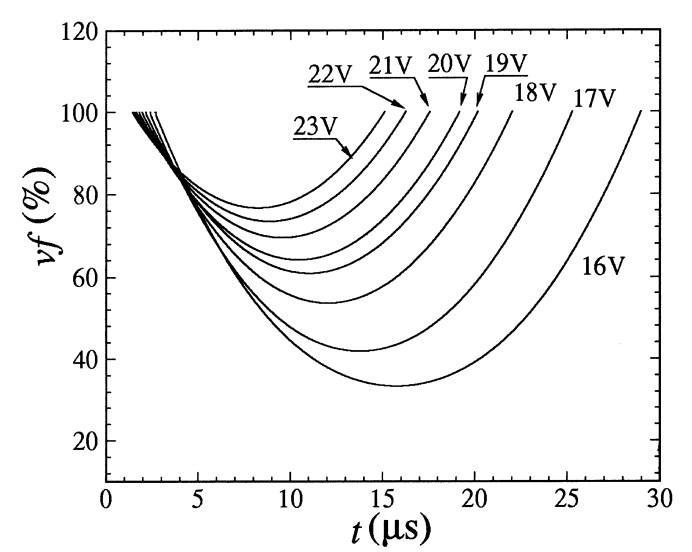

(b)

Fig. 3. Effect of operation voltage of electrical pulse on the predicted results from the one-dimensional model in Chen et al. (1997a) at $W=3 \mu$ s: (a) pressure response curve; (b) volume percentage of ink in the nozzle.

breaks-off from the nozzle exit, Eq. (12) and Eq. (13) are the only two equations left to be solved for obtaining the local velocity distribution in the ejected ink droplet. The numerical computation continues until the long tail droplet separates from the main ink droplet.

\section{Numerical scheme}

The present study employed the finite-difference scheme to accommodate the growth of ejected ink droplet. The computational grid, which is fine on both ends of ejected ink droplet remains the same number of grid points through the numerical computation. The non-uniform grid on the physical coordinate $(\bar{z}, \bar{t})$ was then transformed into a uniform grid on the computational coordinate $(\zeta, \bar{t})$ which ranges from $\zeta=0$ to $\zeta=1$ for $\bar{t} \geqslant 0$.
In this study, the solutions of Eq. (12) and Eq. (13) were obtained by an implicit finite-difference scheme. Both equations were converted into finite-difference formulations by approximating the time derivative with first-order forwarddifference and the spatial derivative with second-order central-difference. Thus, the present numerical scheme has first-order accuracy in time domain and second-order accuracy in space.

Once the velocity field and meniscus radius of ink droplet were obtained, the leading and trailing edges of droplet should be updated for the next time step by the following expressions:

$z_{\mathrm{N}}^{n+1}=z_{\mathrm{N}}^{n}+V_{\mathrm{N}}^{n} \Delta t$

and

$z_{1}^{n+1}=z_{1}^{n}$, before the break-off of droplet,

$z_{1}^{n+1}=z_{1}^{n}+V_{1}^{n} \Delta t$, after the break-off of droplet,

where subscripts 1 and $\mathrm{N}$ denote the nodal index of trailing and leading edges of droplet, respectively. Note that the trailing edge of droplet was fixed at the nozzle exit before the break-off of droplet from the nozzle. As both new locations of trailing and leading edges were known, the physical domain was remeshed, which became fine near both edges and coarse on the central region of droplet. Then, the guessed values of velocity and meniscus radius were imposed on all grid nodes. With these new guessed values, Eq. (18) and the finite-difference expressions of Eq. (12) and Eq. (13) were solved to obtain the velocity and meniscus radius on all grid nodes. In order to accelerate the iterative procedure, the successive over-relaxation technique is used to guess the new values in both velocity and meniscus radius. The iterative procedure was continued until the relative residual between two successive iterations for all nodal points was less than $10^{-4}$. Break-off time of the ejected ink droplet from nozzle exit and the separation time of long tail from the main droplet are taken numerically at which the local meniscus radius drops below $0.05 a$, respectively.

For checking the dependence of predicted results on the number of grid nodes, two different numbers of grid node were tested in this study. Predicted results are listed in Table 1. The maximum difference on the predicted results was less than $0.5 \%$ as the number of nodes increased from 107 to 192 for $t \leqslant 60 \mu \mathrm{s}$. Thus, a computational mesh with 192 nodes was used throughout the present study with time increment of $0.01 \mu \mathrm{s}$.

One should note that there is no ink flow motion before the formation of vapor bubble. Therefore, the leading edge of droplet at the time instant of bubble formation, $t=t_{\mathrm{b}}$, was determined simply by solving Eq. (18) by assuming the meniscus radius to be infinite. After the vapor bubble formation, the set of three equations Eqs. (12), (13) and (18) should be solved to predict the velocity and meniscus radius of droplet.

\section{Results and discussion}

Effect of gravity on the predicted results of ink droplet was investigated. At $E=20 \mathrm{~V}, W=3 \mu$ sith $\mathrm{We} / \mathrm{Re}=0.0385$, Table 2 lists the numerical results of $z_{\text {tip }}$ at various time instants,

Table 1

Grid dependence test on the predicted results at $E=17 \mathrm{~V}$ and $W=3 \mu$ s with $\mathrm{We} / \mathrm{Re}=0.0385$, and $\mathrm{Fr}=84.85$

\begin{tabular}{llllll}
\hline Number of grid nodes & $z_{\text {tip }}, t=10 \mu \mathrm{s}$ & $z_{\text {tip }}, t=30 \mu \mathrm{s}$ & $z_{\text {tip }}, t=50 \mu \mathrm{s}$ & $t_{\text {break }}$ & $t_{\mathrm{s}}$ \\
\hline 107 grid & $120.80 \mu \mathrm{m}$ & $412.02 \mu \mathrm{m}$ & $689.40 \mu \mathrm{m}$ & $24.74 \mu \mathrm{s}$ & $64.40 \mu \mathrm{s}$ \\
192 grid & $121.39 \mu \mathrm{m}$ & $412.72 \mu \mathrm{m}$ & $690.47 \mu \mathrm{m}$ & $24.70 \mu \mathrm{s}$ & $64.50 \mu \mathrm{s}$ \\
\hline
\end{tabular}


Table 2

Effect of gravity on the predicted results of $z_{\text {tip }}$ at various time instants, $t_{\text {break }}$, and $t_{\mathrm{s}}$ at $E=20 \mathrm{~V}$ and $W=3 \mu \mathrm{s}$ with We/Re $=0.0385$

\begin{tabular}{llllll}
\hline $\operatorname{Fr}$ & $z_{\text {tip }}, t=10 \mu \mathrm{s}$ & $z_{\text {tip }}, t=30 \mu \mathrm{s}$ & $z_{\text {tip }}, t=50 \mu \mathrm{s}$ & $t_{\text {break }}$ & $t_{\mathrm{s}}$ \\
\hline 84.85 & $113.3775 \mu \mathrm{m}$ & $357.2650 \mu \mathrm{m}$ & $579.4792 \mu \mathrm{m}$ & $22.93 \mu \mathrm{s}$ & $57.40 \mu \mathrm{s}$ \\
$\infty$ & $113.3754 \mu \mathrm{m}$ & $357.2636 \mu \mathrm{m}$ & $579.4696 \mu \mathrm{m}$ & $22.93 \mu \mathrm{s}$ & $57.41 \mu \mathrm{s}$ \\
\hline
\end{tabular}

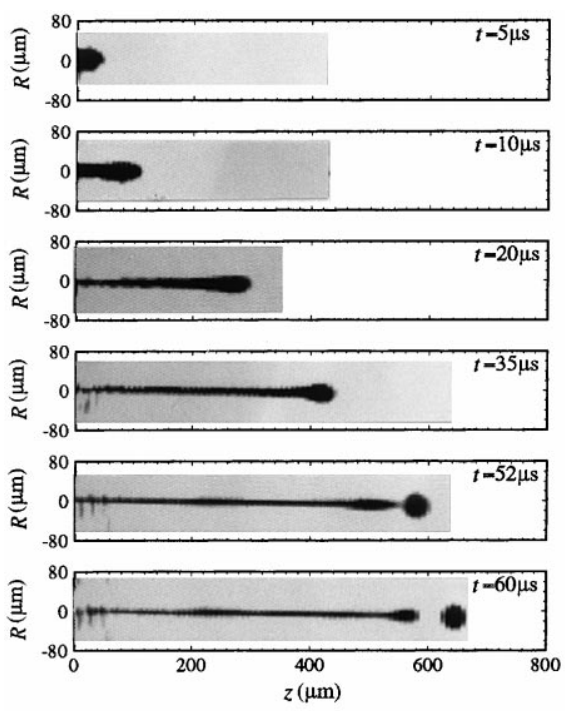

(a)

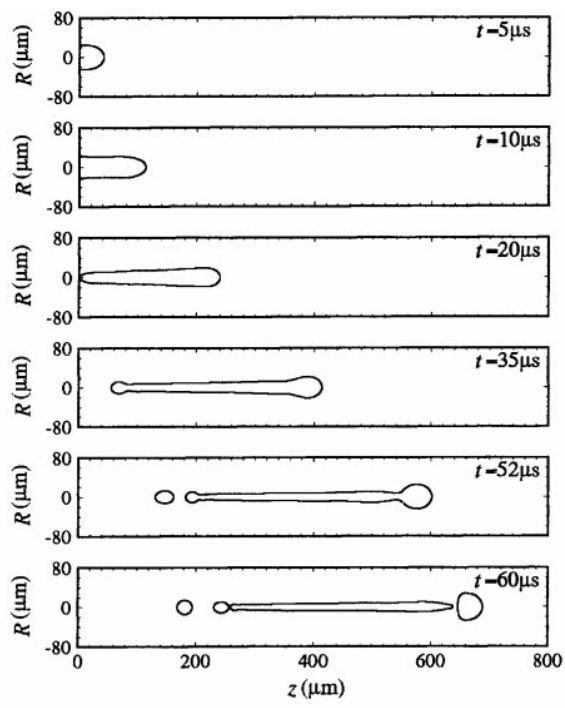

(b)

Fig. 4. Comparison on the growth of ejected ink droplet between (a) experimental results of Chen et al. (1997b) and (b) numerical results of present study at $E=20 \mathrm{~V}$ and $W=3 \mu$ s with $\mathrm{We} / \mathrm{Re}=0.0385$.

$t_{\text {break }}$, and $t_{\mathrm{s}}$ by Froude numbers of $\infty$ and 84.85. Both values of $\mathrm{We} / \mathrm{Re}=0.0385$ and $\mathrm{Fr}=84.85$ were chosen to match the physical properties and dimensions of the present test printhead and ink. With $\mathrm{Fr}=\infty$, the gravity was neglected. It can be observed that little effect of gravity on the droplet formation process has been found. Therefore, the gravity was neglected in the results presented thereafter.

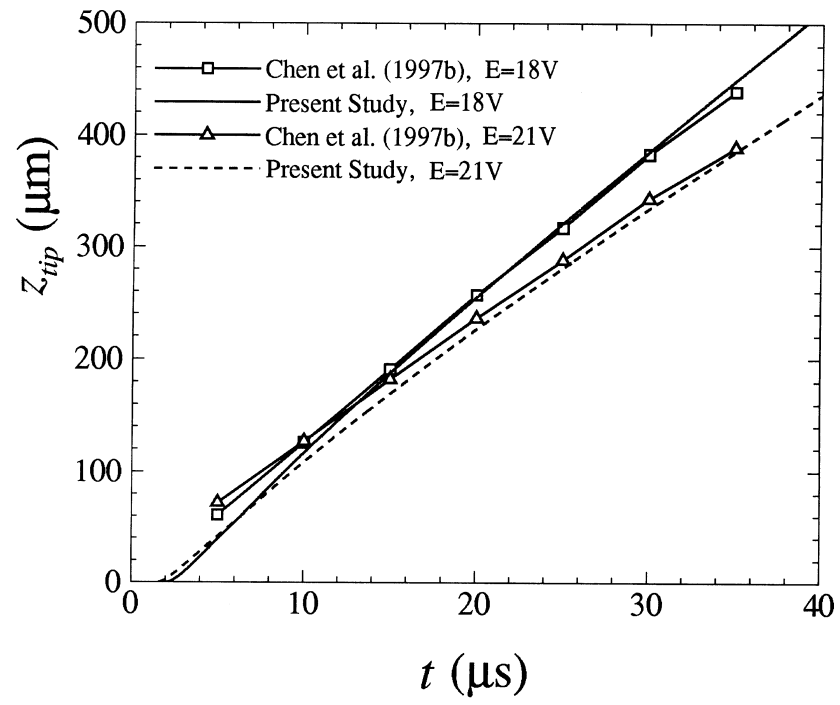

Fig. 5. Comparison on $z_{\text {tip }}$ with respect to time between experimental results of Chen et al. (1997b) and numerical results of present study with $\mathrm{We} / \mathrm{Re}=0.0385$ at $E=18 \mathrm{~V}$ and $E=21 \mathrm{~V}$, respectively.
Figs. 4-6 show the comparisons between the present numerical predictions and flow visualization results by Chen et al. (1997b) on the droplet formation process, ejected droplet length, and separation time of the long tail from the main droplet respectively, at $W=3 \mu \mathrm{s}$ with $\mathrm{We} / \mathrm{Re}=0.0385$. At $E=20 \mathrm{~V}$, the predicted droplet formation process well agrees with the flow visualization results except the size of the satellite

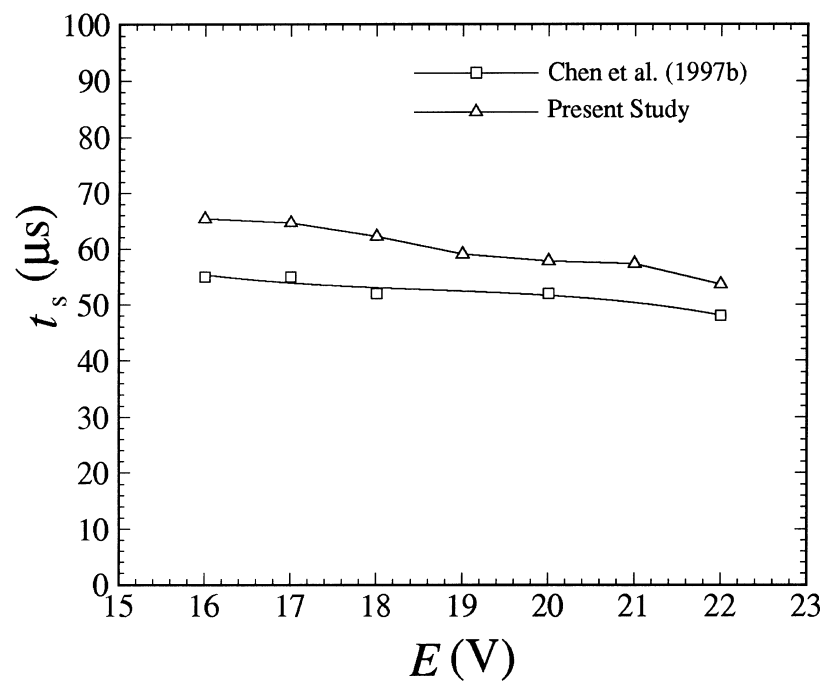

Fig. 6. Comparison on $t_{\mathrm{s}}$ under various operating voltages between experimental results of Chen et al. (1997b) and numerical results of present study at $W=3 \mu$ s with $\mathrm{We} / \mathrm{Re}=0.0385$. 
droplet. Due to a large pressure difference between the vapor bubble and the atmosphere, the leading edge of droplet moves in the air with a velocity greater than $10 \mathrm{~m} / \mathrm{s}$ at $W=3 \mu \mathrm{s}$, $E=20 \mathrm{~V}$ with $\mathrm{We} / \mathrm{Re}=0.0385$. Since the ink is driven out of the nozzle with linear momentum which is large during the bubble growth and small during the shrinking of bubble, a large velocity variation occurs in the ejected ink. These variations of velocity and capillary force can result in the comet shape of droplet, a round leading edge with a slender and long tail. Predicted results illustrate the break-off of droplet from the nozzle near a time instant of $20 \mu$ s which is earlier than the flow visualization result. This is possibly due to the simplification of the two-dimensional momentum equation in cylindrical form into a one-dimensional model. During the travel of droplet in the air, a satellite droplet separates from the trailing edge of main droplet that follows by the appearance of second satellite droplet and the separation of the long tail from the main droplet. As expected, the velocity of separated satellite droplet is significantly less than that of main droplet because the trailing part of droplet is out of the nozzle with a lower linear momentum than that of leading part. At the final stage of traveling in air, the droplet can break into several parts including a main droplet, a long tail, and two satellite droplets. This interesting droplet formation process is caused by the large momentum variation of ejected ink at the nozzle exit and the large capillary force of meniscus with radii in few micrometers. The impingement of separate tail and satellite droplets on the paper can result in blurs on the paper surface and severely affect the printing quality of a printhead. In addition, the measured results in Fig. 4 show that the ink droplet has already broken from the nozzle exit near the time of $60 \mu \mathrm{s}$. This indicates the gap between paper and printhead should be greater than $650 \mu \mathrm{m}$ for the present test operating condition, otherwise the droplet will be torn apart by the relative movement between the printhead and paper but not by the axial motion of ink droplet. Thus, ink might be destined to the locations which should be left to be blank.

Fig. 5 also shows the effect of operating voltage on the distance from the leading edge of droplet to the nozzle exit at $W=3 \mu$ s with $\mathrm{We} / \mathrm{Re}=0.0385$. The traveling distances of leading edge of droplet increases almost linearly with time. This indicates the viscous force exerted by the surrounding air on the droplet has little effect on the flying speed of droplet. Thus, the leading edge velocity of droplet can be estimated from measured distances. It can be clearly observed that the leading edge velocity is lower for a higher operating voltage. This is due to the fact that earlier pressure drop occurs in the vapor bubble for a higher operating voltage, as shown by the pressure response curves in Fig. 3(a). A good agreement between experimental and numerical results can be found in Fig. 5 .

An approximate difference of $20 \%$ is observed between the predicted and flow visualized data on the separation time of long tail from the main droplet as shown in Fig. 6. The flow visualization results were recorded every $\mu$ s after the application of electrical impulse. This difference is possibly due to the omission of radial velocity in the ink droplet in present study.

Effect of the operating voltage on the evolving droplet formation is shown in Fig. 7 with $\mathrm{We} / \mathrm{Re}=0.0385$. Droplet formation process is quite similar for both operating voltages of 17 and $22 \mathrm{~V}$. The lower operating voltage produces a larger main droplet, a longer tail, and a later separation of the long tail from the main droplet. These phenomena are caused by a greater axial variation in the linear momentum of ejected ink with a lower operating voltage. Fig. 8 shows the effect of operating voltage on the break-off time of ink droplet from the nozzle exit. The break-off time of droplet decreases almost linearly with an increase in the operating voltage. As expected, a later formation of vapor bubble and a longer period of appearance of bubble prolongs the duration of ejecting ink with a positive pressure force which will drive more ink out of the nozzle with a low operating voltage. As shown in Fig. 3(b), the ejection of ink out of the nozzle stops near $t=13 \mu$ s at $E=17 \mathrm{~V}$ but near a much earlier time of $9 \mu$ s at $E=22 \mathrm{~V}$. Without a continuous supply of ink or even worse with a reverse ink motion in the nozzle due to the shrinkage of bubble, the ejected droplet breaks-off from the nozzle exit.

Fig. 9 shows the droplet formation process with two different $\mathrm{We} / \mathrm{Re}$ ratios of 0.1 and 0.4 at $E=20 \mathrm{~V}$ and $\mathrm{W}=3 \mu \mathrm{s}$. Higher We/Re ratio implies a lower surface tension coefficient or a higher viscosity of ink, if the density of ink and the exit diameter of nozzle are fixed. The predicted results show that a higher $\mathrm{We} / \mathrm{Re}$ ratio results in a shorter droplet with a lower linear momentum. It is expected that the higher viscosity
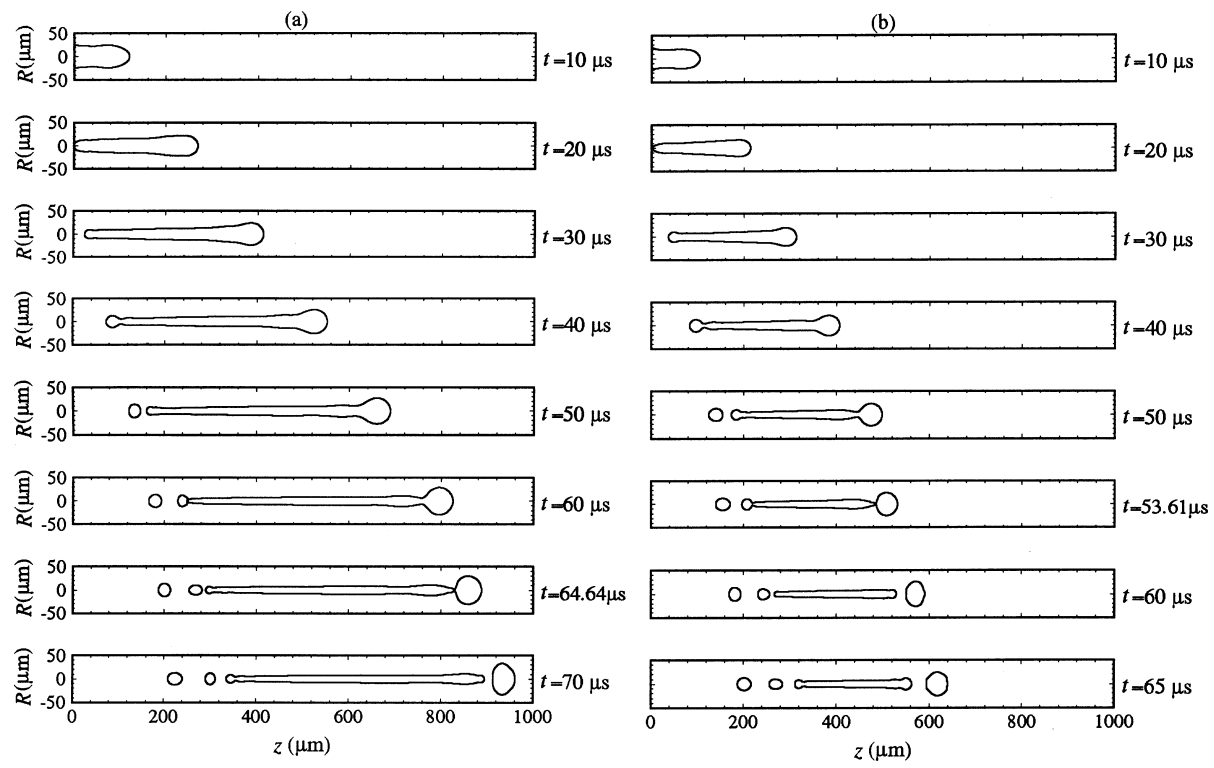

Fig. 7. The growth of ejected ink droplet with $\mathrm{We} / \mathrm{Re}=0.0385$ at $W=3 \mu$ s and the operating voltage of (a) $E=17 \mathrm{~V}$; (b) $E=22 \mathrm{~V}$. 


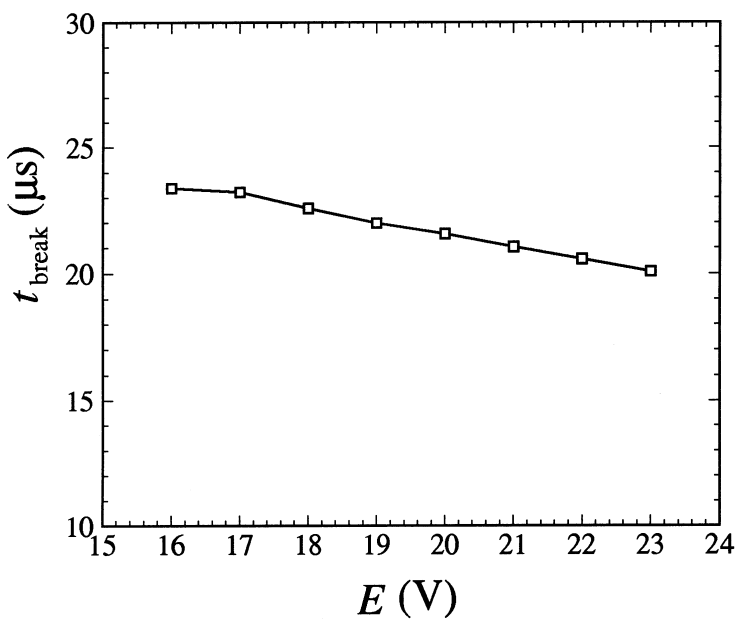

Fig. 8. Effect of operating voltage on predicted $t_{\text {break }}$ at $W=3 \mu$ s with $\mathrm{We} / \mathrm{Re}=0.0385$.

reduces the flying speed of ink droplet. In addition, the separation of the first satellite droplet occurs earlier for the ink with a lower We/Re ratio, that is, with a larger surface tension coefficient or a lower viscosity. A much greater pressure difference across the meniscus can occur near both neck regions of droplets for the ink with a greater surface tension than that with a lower surface tension. Such a great pressure difference causes the earlier separation of satellite droplets from the long and slim tail. The same reason can explain why the ink droplet breaks-off later from the nozzle with a higher We/Re value. It can be clearly observed from Fig. 9(b) that no single main droplet is formed even a relatively long time, $t=89.58 \mu \mathrm{s}$, has elapsed after the electrical pulse was applied to the resistor layer with $\mathrm{We} / \mathrm{Re}=0.4$. Therefore, it becomes more difficult to control the printing quality for allowing such a long droplet to strike on the paper surface with $\mathrm{We} / \mathrm{Re}=0.4$. Figs. 10 and 11 show the effect of $\mathrm{We} / \mathrm{Re}$ ratio on the break-off time of ink droplet from the nozzle and on the separation time of long tail from the main droplet respectively. Both values of $t_{\text {break }}$ and $t_{\mathrm{s}}$

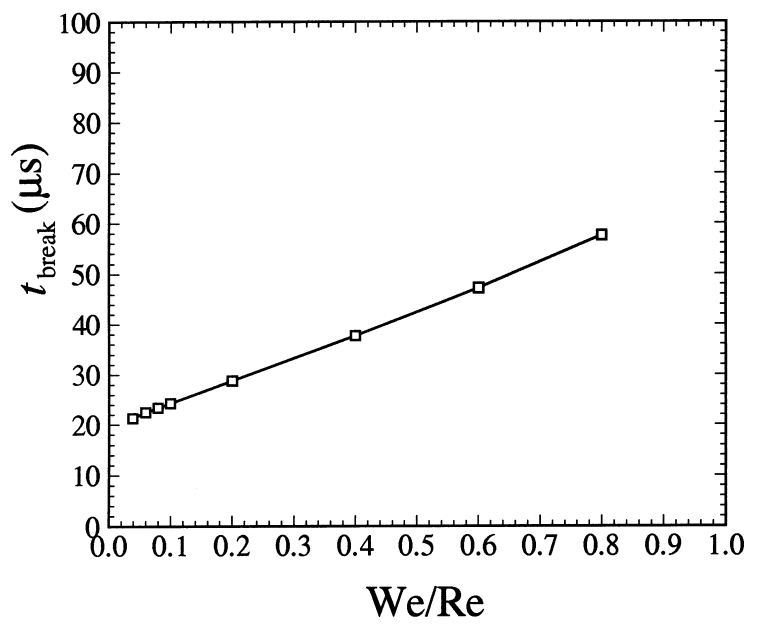

Fig. 10. Effect of We/Re ratio on predicted $t_{\text {break }}$ at $E=20 \mathrm{~V}$ and $W=3$ $\mu \mathrm{s}$.

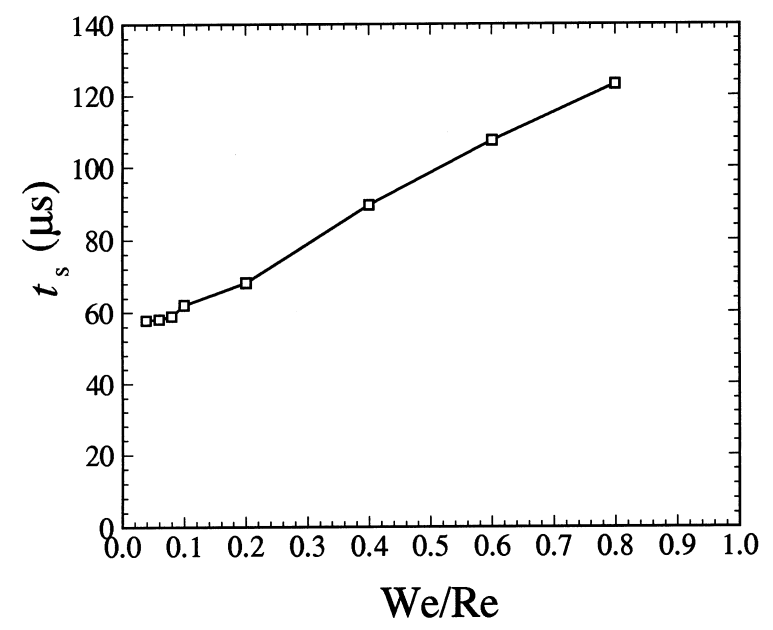

Fig. 11. Effect of We/Re ratio on predicted $t_{\mathrm{s}}$ at $E=20 \mathrm{~V}$ and $W=3 \mu \mathrm{s}$.
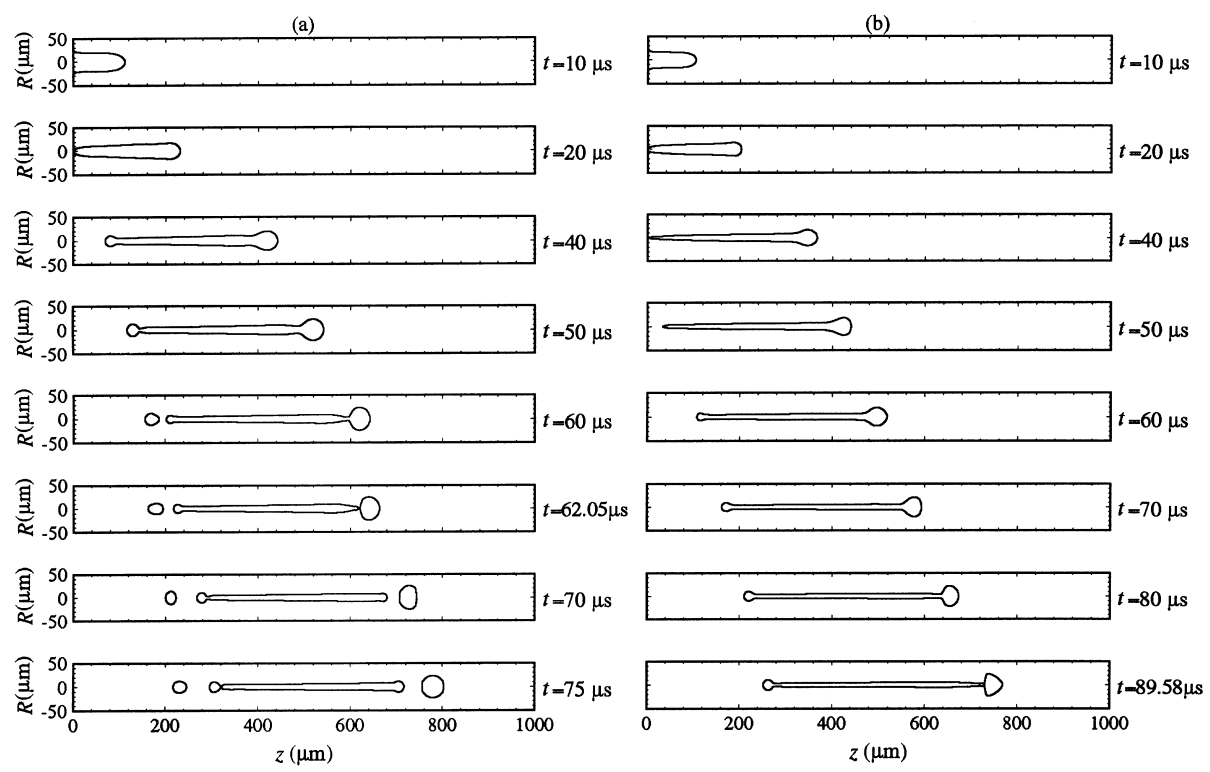

Fig. 9. The growth of ejected ink droplet at $E=20 \mathrm{~V}$ with (a) $\mathrm{We} / \mathrm{Re}=0.1$; (b) $\mathrm{We} / \mathrm{Re}=0.4$. 
increase almost linearly with $\mathrm{We} / \mathrm{Re}$ ratio. That is, a lower surface tension coefficient or a greater viscosity of ink can delay the break-off or separation of the ink droplet, which should be avoided for a well-designed printhead.

\section{Conclusions}

The complete droplet formation process of a sideshooter thermal bubble printhead is simulated in this study. The local ink velocity in the ejected droplet was obtained by using an implicit finite-difference scheme to solve a simplified one-dimensional model. Numerical results are presented to show the pressure response curves in the vapor bubble after homogeneous nucleation in the ink, the droplet formation process, the break-off and separation time instants of ink droplets. Effects of gravity, ink property, and operating voltage of electrical pulse on the droplet formation process were also investigated.

Numerical results show that the droplet formation is not much affected by the gravitational force due to the extreme pressure difference between the vapor bubble pressure and atmospheric pressure. Despite of the simplicity of one-dimensional model solved in this study, comparisons between the flow visualization results in an earlier study (Chen et al., 1997b) and the present predictions show good agreements among the evolving droplet formation process and the flying distances of droplet leading edge from the nozzle exit. This good agreement assures that the present numerical method can be a useful tool to the design of nozzle shape, choose of ink, and also definition of the operating condition for a sideshooter thermal bubble printhead to produce the desired ink droplet with a correct shape and volume.

The ejected droplet will have a greater droplet volume, a higher linear momentum, a later break-off time from the nozzle exit, and a longer droplet length by reducing the operating voltage. However, the ink droplet owns a longer droplet length with greater linear momentum and an earlier break-off time of droplet from the nozzle exit by lowering the ratio between the viscous and capillary forces, that is, a lower $\mathrm{We} / \mathrm{Re}$ value.

\section{Acknowledgements}

The authors deeply appreciate the financial support by NSC under the grant number of NSC 86-2212-E002-080 for carrying out this study.

\section{References}

Adams, R.L., Roy, J., 1986. A one-dimensional numerical model of a drop-on-demand ink jet. J. Appl. Mech. 53, 193-197.

Asai, A., Hara, T., Endo, I., 1987. One-dimensional model of bubble growth and liquid flow in bubble jet printers. Jpn. J. Appl. Phys. 26, 1794-1801.

Asai, A., 1991. Bubble dynamics in boiling under high heat flux pulse heating. ASME J. Heat Trans. 113, 973-979.

Asai, A., 1992. Three-dimensional calculation of bubble growth and drop ejection in a bubble jet printer. ASME J. Fluids Eng. 114, 638-641.

Chen, P.H., Chen, W.C., Chang, S.H., 1997a. Bubble growth and ink ejection process of a thermal ink jet printhead. Int. J. Mech. Sci. 39, 683-695.

Chen, P.H., Chen, W.C., Chang, S.H., 1997b. Visualization of drop ejection process of a thermal bubble ink jet printhead. Proceedings of the First Pacific Symposium on Flow Visualization and Image, vol. 1, pp. 132-137.

Fromm, J.E., 1984. Numerical calculation of the fluid dynamics of drop-on-demand jets. IBM J. Res. Develop. 28, 323-333.

Krause, P., Obermeier, E., Wehl, W., 1996. A micromachined singlechip inkjet printhead. Sensors and Actuators A 53, 405-409. 